\title{
SELECTIVE RESPONSE OF DOPAMINE IN THE PRESENCE OF ASCORBIC ACID AND URIC ACID AT GOLD NANOPARTICLES AND MULTI-WALLED CARBON NANOTUBES GRAFTED WITH ETHYLENE DIAMINE TETRAACETIC ACID MODIFIED ELECTRODE
}

\author{
Chun-ran Tang ${ }^{1,2^{*}}$, Ge Tian ${ }^{1}$, Ya-jing Wang ${ }^{1}$, Zhong-hua Su ${ }^{1}$, Chun-xiang Li ${ }^{1}$, Bao-gang Lin ${ }^{1}$,
} Hao-wen Huang ${ }^{1}$, Xian-yong $\mathrm{Yu}^{1}$, Xiao-fang $\mathrm{Li}^{1}$, Yun-fei Long ${ }^{1}$ and Yun-long Zeng ${ }^{1,2 *}$

${ }^{1}$ Key Laboratory of Theoretical Chemistry and Molecular Simulation of Ministry of Education, School of Chemistry and Chemical Engineering, Hunan University of Science and Technology, Hunan Xiangtan 411201, PR China

${ }^{2}$ State Key Laboratory of Chemo/Biosensing and Chemometrics, Hunan University, Changsha 410082, PR China

(Received October 2, 2008; revised May 13, 2009)

\begin{abstract}
By grafting with ethylene diamine tetraacetic acid (EDTA), novel multi-walled carbon nanotube nano-composites (EDTA-MWCNTs) have been successfully prepared. The integration of gold nanoparticles (AuNPs) and novel functionalized matrix shows very promising applications in the fabrication of biosensors for sensitive detection of dopamine in the presence of ascorbic acid and uric acid with the combination of the charge repelling property of carboxylic anion and the electrocatalytic effect of EDTA. The AuNPs and EDTA-MWCNTs modified film shows excellent sensitivity and selectivity for the dopamine analysis due to the synergic effect of AuNPs and EDTA-MWCNTs. As high as 2000 fold and 150 fold acceptable tolerance of ascorbic acid and uric acid for the determination of trace dopamine is reached, respectively. The linear concentration range for dopamine is from $1.0 \times 10^{-8}$ to $6.0 \times 10^{-6} \mathrm{~mol} . \mathrm{L}^{-1}$, and the detection limit is $3.0 \times 10^{-9} \mathrm{~mol} . \mathrm{L}^{-1}$. The response of the sensor is very quick and response time is less than $1 \mathrm{~s}$. The method can be applied to detect the dopamine in human urine samples.
\end{abstract}

KEY WORDS: Dopamine, Biosensor, Ethylene diamine tetraacetic acid grafting multi-walled carbon nanotubes, Gold nanoparticles, Synergistic effect

\section{INTRODUCTION}

Design and development of a simple and rapid detection method for dopamine (DA), an important neurotransmitter in mammalian central nervous system [1, 2] has drawn much attention [3-6]. In real biological matrices, the concentration of DA is very low, while many coexisting substances such as ascorbic acid (AA) are in relatively high, 1000 times higher than that of DA generally. In addition, the oxidation potential of AA, DA and uric acid (UA) are close to each other. Thus, the determination of DA in the presence of high level of AA and UA in real biological samples faces a big challenge in improvement both selectivity and sensitivity. Therefore, the crucial problem in dopamine sensor design is removing the interference caused by AA and UA. It is known that $\mathrm{AA}\left(\mathrm{pK}_{\mathrm{a}} 4.10\right)$ and UA $\left(\mathrm{pK}_{\mathrm{a}} 5.75\right)$ exist in their anionic form while DA is in its cationic form $\left(\mathrm{pK}_{\mathrm{b}} 8.87\right)$ at the physiological $\mathrm{pH}$ of 7.4. Thus, the working electrode modified with anionic polymer films is widely used to reduce the AA interference in the electroanalysis of DA [7-9]. Recently research developed an excellent permselectivity of the combined tyramine and pyrrole-1-propionic acid (PTy/PPA) film which shows high selective toward dopamine [10]. The properties of the sensor have been improved greatly. The modification of boron-doped diamond electrodes with a combined electropolymerized film of PTy/PPA has allowed detection of dopamine in the presence of dopamine metabolites and excess ascorbic acid.

*Corresponding author. E-mail: yunlongzeng1955@126.com 
Nanoparticles such as carbon nanotubes (CNTs) and gold nanopartlcles (AuNPs) possess high electrocatalytic active, specific electronic properties, can facilitate electron transfer between the electroactive species and electrode, provide a new avenue for fabricating biosensors [11-13]. Many modified electrodes based on CNTs have been widely used for the study of DA, such as CNTs paste modified electrode, single walled carbon nanotube-modified platinum electrode [14], single-walled carbon nanotubes-Fc modified glassy carbon electrode (GCE) [15], Nafion/carbon nanotubes coated poly(3-methylthiophene) modified GCE [16], multi-walled carbon nanotubes (MWCNTs) modified gold electrode [17], layer-by-layer assembled MWCNTs modified electrode [18], etc. In many cases, the sensors based on CNTs or AuNPs the have the detection limit of about $1 \mu \mathrm{mol} . \mathrm{L}^{-1}$ or lower [17, 19-21]. Hence, improving the selectivity and sensitivity of the DA sensor is still a significative research. Recently, composite materials based on integration of CNT and some other materials to possess properties of the individual components with a synergistic effect have gained growing interest. Materials for such purposes include conducting polymers, redox mediators and metal nanoparticles [22-25]. For example, coupling CNT with cobalt hexacyanoferrate nanoparticles (CoNP) resulted in remarkable improvement of the electroactivity of the composite materials toward glucose through synergistic effect [25]. The CoNP-CNT-CHIT/GOx biosensor exhibited linearity from $10 \mu \mathrm{mol} . \mathrm{L}^{-1}$ up to $10 \mathrm{mmol} . \mathrm{L}^{-1}$ with a detection limit of $5 \mu \mathrm{mol} . \mathrm{L}^{-1}$, whereas, the CNT-CHIT and CoNP-CHIT film modified glucose biosensor exhibited very small response toward glucose with a detection limit of $300 \mu \mathrm{mol} . \mathrm{L}^{-1}$ and $1 \mathrm{mmol} . \mathrm{L}^{-1}$ [25], respectively.

In the present research, MWCNTs with the charge repelling property of carboxylic anion were first prepared by grafting ethylene diamine tetraacetic acid (EDTA) on to the surface of multi-walled carbon nanotubes in order to anti-interference of AA and UA. Then, a novel DA sensor was prepared by covalently attracted AuNPs and EDTA-MWCNTs onto the surface of GCE. The properties of the sensor improved significantly benefited from the synergic action of AuNPs and EDTA-MWCNTs.

\section{EXPERIMENTAL}

\section{Reagents}

MWCNTs (95\%, with outer diameters of ca 10-20 nm and lengths of 5-10 mm) were from Shenzhen Nanotech. Port. Co. Ltd. (Shenzhen, China). DA, UA were from Sigma (USA). 3Mercaptopropylmethyldimethoxysilane (MPDeOS), 1-(3-dimethylaminopropyl)-3-ethylcarbodiimide hydrochloride (EDC, 98+\%), N-hydroxysuccinimide (NHS, 98+\%) were sourced from Acros Organics (USA). EDTA and other reagents were of analytical reagent and from Shanghai Chemical Reagents Co (China), and used without further purification. Solutions of AA, DA and UA were freshly prepared before use. Buffers of $\mathrm{Na}_{2} \mathrm{HPO}_{4}-\mathrm{KH}_{2} \mathrm{PO}_{4}\left(\mathrm{PBS}, 0.20\right.$ mol.L $\left.{ }^{-1}\right)$ at various $\mathrm{pH}$ values were prepared and used as the supporting electrolyte. Doubly distilled water was used throughout all experiments.

\section{Apparatus}

Infrared spectra were recorded using FTIR Spectrum One (Perkin Elmer). Electrochemical measurements were performed with a three-electrode system comprising of a platinum foil as the auxiliary electrode, a saturated calomel electrode as reference electrode, and a working electrode. A MPDeOS modified GCE and a EDTA-MWCNTs modified GCE were used as the working electrode. All electrochemical experiments were performed on a LK 98 Microcomputer-based Electrochemical Analyzer (Tianjin, China). 
Procedure

Treatments of glassy carbon electrode and multi-walled carbon nanotubes

Prior to modification, GCE was polished with 3 and $1 \mu \mathrm{m}$ aluminum slurries, the electrode was then rinsed copiously with water and sonicated in a water bath for $2 \mathrm{~min}$, then treated with a solution of $\mathrm{HNO}_{3} / \mathrm{H}_{2} \mathrm{O}(1: 4, \mathrm{v}: \mathrm{v})$ for $30 \mathrm{~s}$. After being rinsed, the electrode was polished with a $0.05 \mu \mathrm{m}$ aluminum slurry to mirror finish. The electrode was rinsed again with water and sonicated for $2 \mathrm{~min}$, finally let it air-dry.

The MWCNTs were pretreated according to the procedure with minor modifications [26, 27]. They were oxidized by refluxing in concentrated $\mathrm{HNO}_{3}$ and $\mathrm{H}_{2} \mathrm{SO}_{4}$ (volume ratio of $1: 3$ ) for $12 \mathrm{~h}$. The resultant mixture was diluted with pure water to about three times of the original volume and stirred for $24 \mathrm{~h}$. The mixture was subjected to high-speed to separate the black solid carboxylated MWCNT powder. After filtrating and washing with pure water several times, the powder was dried in a vacuum at $50{ }^{\circ} \mathrm{C}$. The carboxylated CNTs were then dispersed in water by sonicating them to form an aqueous suspension of $1.0 \mathrm{mg} \mathrm{mL}^{-1}$ for further functionalization. An aqueous suspension $(50 \mathrm{~mL})$ of $0.1 \mathrm{mg} \mathrm{mL}^{-1}$ carboxylated MWCNT containing $100 \mathrm{mg}$ of EDC and $100 \mathrm{mg}$ of NHS was slowly added into an aqueous solution $(1.5 \mathrm{~mL}$ of $0.2 \%$ ethylene-diamine under high-speed agitating. The black slimy deposition of ethylene-diamine modified MWCNT was obtained via centrifugal sedimentation and washing. Then the deposition was dispersed in $50 \mathrm{~mL}$ water again by sonicated. The aqueous suspension $(50 \mathrm{~mL})$ of ethylene-diamine modified MWCNT prepared above containing $100 \mathrm{mg}$ of EDC and $100 \mathrm{mg}$ of NHS was slowly added into an aqueous solution $(1.5 \mathrm{~mL})$ of $1 \%$ EDTA. EDTA-MWCNT was obtained by purifying with centrifugal sedimentation and washing with pure water to remove the excessive EDTA and auxiliary reagents.

\section{Preparation of Au nanoparticles}

All glassware used in the experiment was cleaned in a bath of freshly prepared $3: 1 \mathrm{HCl} / \mathrm{HNO}_{3}$ and rinsed thoroughly in water prior to use. AuNPs were prepared according to the literature report [28, 29] method. Briefly, a $250 \mathrm{~mL}$ aqueous solution of $0.01 \% \mathrm{HAuCl}_{4}$ was heated to boiling with vigorous stirring, and then $1.9 \mathrm{~mL}$ of $1 \%$ sodium citrate was added rapidly. After the solution change to red-violet, boiling continued for an additional $15 \mathrm{~min}$, the heating source was removed, and the solution was stirred for another $15 \mathrm{~min}$. The red-violet solution was stored at $4{ }^{\circ} \mathrm{C}$ in dark. The solution was subjected to high-speed centrifugal sedimentation to separate the red-violet solid AuNPs. After filtrating and washing with pure water several times, the red-violet solid AuNPs were then dispersed in water by sonicating for modification of GC electrode.

\section{Preparation of the sensors}

For GCE modification, first the glassy carbon electrode was dipped in 0.5\% MPDeOS ethanol solution for $24 \mathrm{~h}$, the electrode was then rinsed copiously with ethanol and sonicated in a ethanol bath for $10 \mathrm{~min}$, the MPDeOS modified electrode was prepared. The MPDeOS modified electrode was immersed into the AuNPs for $12 \mathrm{~h}$ and rinsed copiously with water and sonicated several times in water, and the electrode was then putted into an aqueous solution of $0.1 \%$ cystine for $12 \mathrm{~h}$ and rinsed and sonicated in water several times. After the electrode was dipped into an aqueous saturated solution of EDTA-MWCNT contained $0.1 \%$ EDC and NHS for $12 \mathrm{~h}$, and then rinsed with water. The AuNPs and EDTA-MWCNT modified biosensor was prepared and stored in 0.001 M PBS for further application. 
Human urine analysis

Freshly collected human urine samples were filtered and sequentially diluted in two steps (10fold dilution each) with 0.20 mol.L $\mathrm{L}^{-1}$ PBS ( $\mathrm{pH}$ 7.2) to obtain a final sample of 100-fold dilution. The DA in the prepared samples was determined using the biosenser.

\section{RESULTS AND DISCUSSION}

IR spectrum feature of EDTA-MWCNTs

Figure 1 depicts the FTIR spectra of the rough and chemical modified MWCNTs. The chemical modification of MWCNTs was first introduced carboxylic groups on the surface of the multiwalled carbon nanotubes oxidized by refluxing in concentrated $\mathrm{HNO}_{3}$ and $\mathrm{H}_{2} \mathrm{SO}_{4}$. As shown in Figure $1 \mathrm{~b}$, three new infrared vibration absorption bands at $1600-1700 \mathrm{~cm}^{-1}$ are observed, as the carbonyl and carboxyl groups appear on the surface of the MWCNTs during the process of treatment with the mixed concentrated acids as compared with curve a in Figure 1. Since the reaction between amino groups in ethylenediamine and carboxylic groups in carboxylatedMWCNT forms amide-MWCNT, one can see from the infrared absorption spectrum in Figure $1 \mathrm{c}$ that the absorption peaks at 1640 and $1550 \mathrm{~cm}^{-1}$ increase obviously. These absorption peaks are characteristic amide I and II bands that arise from amide groups in ethylenediamine in the MWCNTs. Figure 1d shows the strong absorption peak at $1705 \mathrm{~cm}^{-1}$ because there are plenty of carboxylic groups at MWCNT surface when EDTA was grafted on the surface of multi-walled carbon nanotubes. The procedure can simply show in Scheme 1.

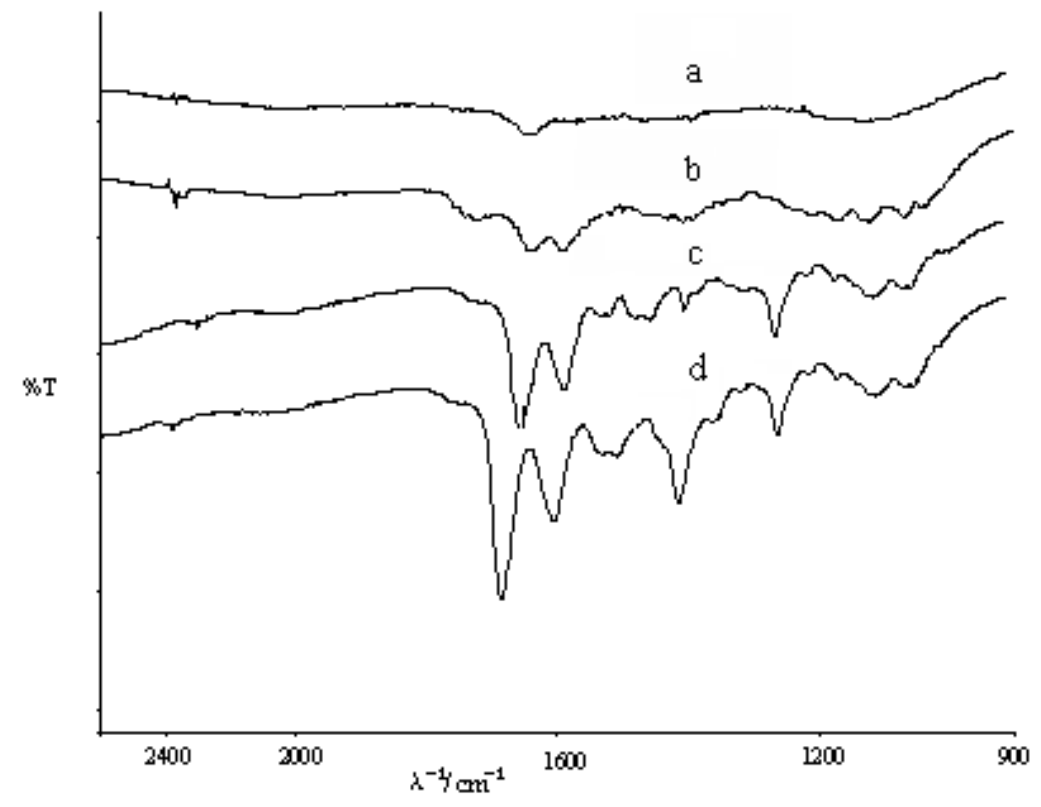

Figure 1. FTIR spectra for (a) rough multi-walled nanotubes (MWCNTs), (b) MWCNTs oxidized by refluxing in concentrated $\mathrm{HNO}_{3}$ and $\mathrm{H}_{2} \mathrm{SO}_{4}$, (c) ethylenediamine modified MWCNTs and (d) ethylene diamine tetraacetic acid (EDTA) grafting MWCNTs.

Bull. Chem. Soc. Ethiop. 2009, 23(3) 


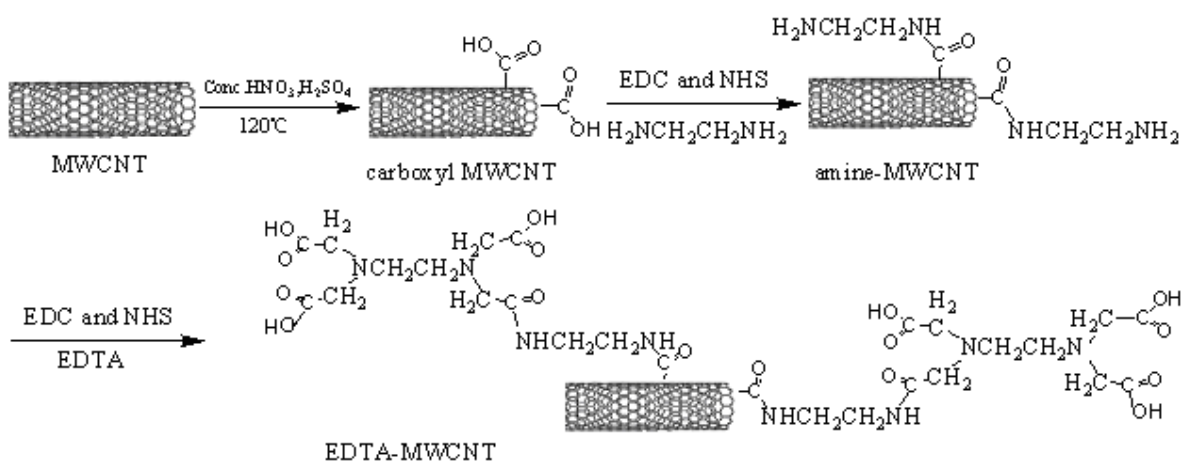

Scheme 1. Process of chemical modification of multi-walled carbon nanotubes with EDTA.

Electrocatalytic oxidation of DA

Figure 2 shows the cyclic voltammograms (CVs) of DA at the GCE electrode modified with EDTA-MWCNTs. As seen in Figure 2a, only very weak current was recorded at MPDeOS modified GCE electrode. A couple of sharp and clear redox peaks of DA are observed in Figure $2 \mathrm{~b}$, which shows a reversible reaction on the EDTA-MWCNT modified biosensor. These phenomena indicate that the EDTA-MWCNT shows high catalytic activity to DA.

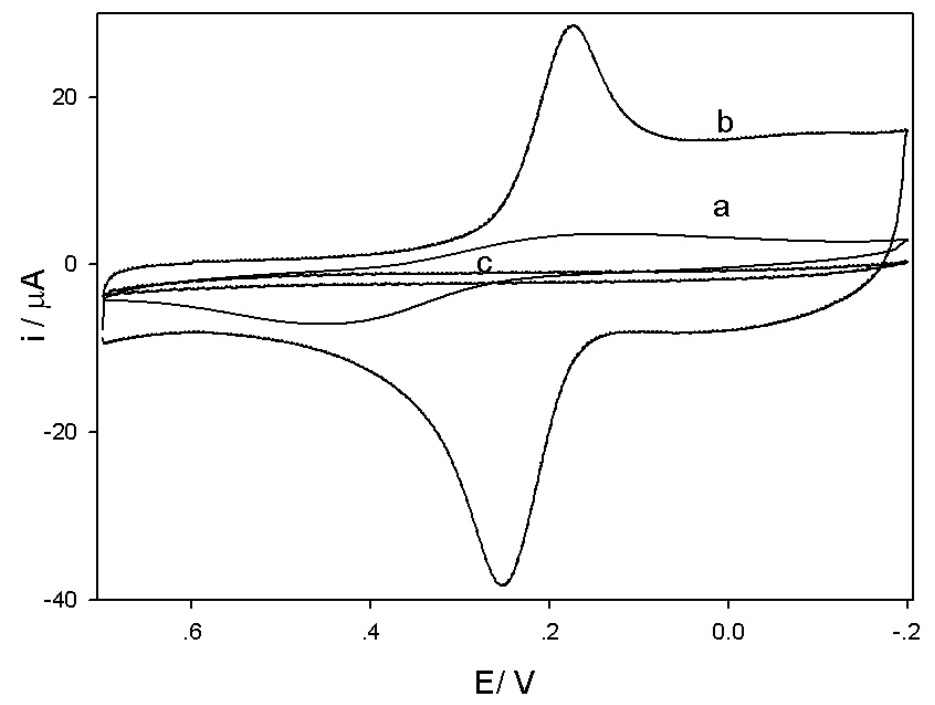

Figure 2. Cyclic voltammograms of the GCE electrodes modified with (a) MPDeOS and (b) AuNPs and EDTA-MWCNTs in $5.0 \mu \mathrm{mol} . \mathrm{L}^{-1}$ dopamine, respectively, and (c) AuNPs and EDTA-MWCNTs in the absence of dopamine at $\mathrm{pH} 7.2 \mathrm{PBS}$. Scan rate: 100 $\mathrm{mV} . \mathrm{s}^{-1}$.

Figure 3 depicts typical cyclic voltammograms of AuNPs and EDTA-MWCNT modified sensor in 0.1 mol. $\mathrm{L}^{-1} \mathrm{PBS}\left(\mathrm{pH} 7.2\right.$ ) containing $5.0 \mu \mathrm{mol} . \mathrm{L}^{-1} \mathrm{DA}$ at different scan rates.

Bull. Chem. Soc. Ethiop. 2009, 23(3) 


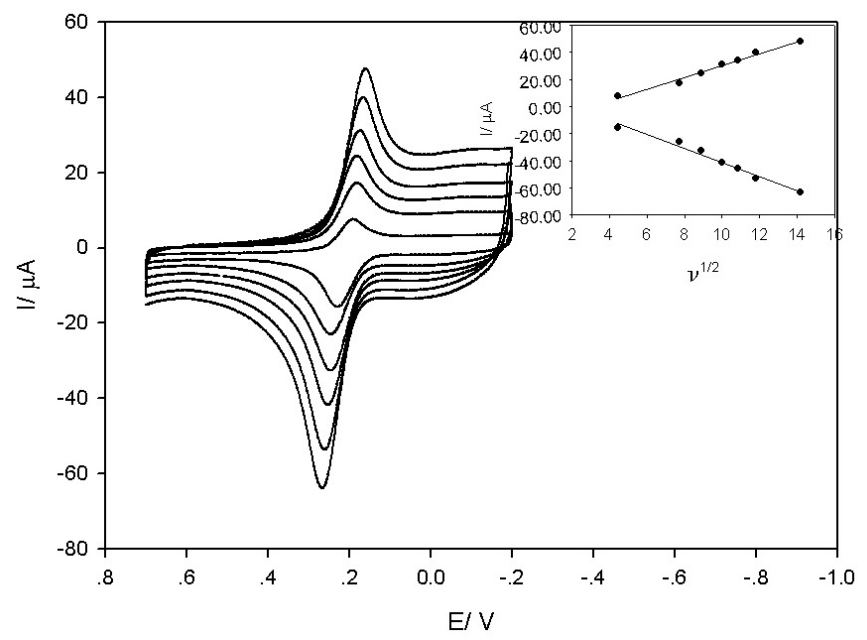

Figure 3. CVs of AuNPs and EDTA-MWCNTs modified GC electrodes in phosphate buffer with $5.0 \mu \mathrm{mol} . \mathrm{L}^{-1}$ dopamine at different scan rates. From inside to outside: 20, 60, 80, $100,120,140$ and $200 \mathrm{mV} . \mathrm{s}^{-1}$. Inset: The anodic and cathodic current plotted against the square root of the scan rate.

The linear relationship between the reduction-oxidation peak current and the square root of scan rate $\left(v^{1 / 2}\right)$ (Figure 3, inset) indicates that the peak current is diffusion-controlled. Thus the oxidized and reduced forms of DA in the solution are not adsorbed on modified electrode surface.

It is known that DA is catalytically oxidized to form $o$-quinone at the electrode [30]:

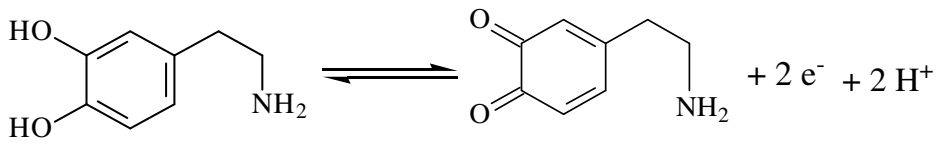

The AuNPs and EDTA-MWCNT modified film shows high electrocatalystic activity toward DA and a couple of sharp and symmetrical redox peaks was observed as seen from Figure 3. The current response curve of the sensor was similar as that of cobalt(II) tetrakisphenylporphyrin modified film [5] toward DA.

\section{Optimization of experimental parameters of the DA electrode}

The biosensor response to DA is influenced by the $\mathrm{pH}$ of background electrolyte and the applied potential. The response current increased with increasing $\mathrm{pH}$ value from 3.0 to 7.2 then decreased as $\mathrm{pH}$ increases further, as show in Figure 4. The maximum response occurred at $\mathrm{pH}$ 7.2 which was chosen for the following experiments.

The influence of working potential on the current response of the biosensor for detection of DA was investigated from 0.150 to $0.400 \mathrm{~V}$. The sensor showed an improved sensitivity with increasing applied oxidation potential. However, as the oxidation potential was over than 0.30 $\mathrm{V}$, the response became unstable. Therefore, the oxidation potential of $0.280 \mathrm{~V}$ was selected for the amperometric measurements in the present study. 


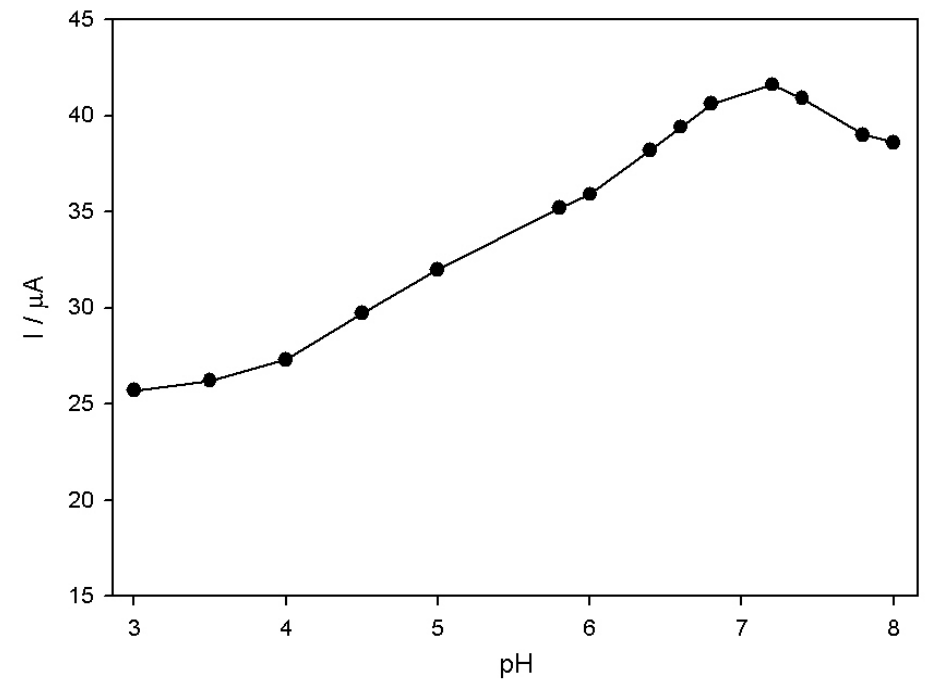

Figure 4. Dependence of current response of sensor modified with AuNPs and EDTAMWCNTs on $\mathrm{pH}\left(0.10\right.$ mol. $\mathrm{L}^{-1} \mathrm{PBS}$ solution $)$ in $1.25 \mu \mathrm{mol} . \mathrm{L}^{-1}$ dopamine, working potential: $0.280 \mathrm{~V}$.

Electrode response characteristics and calibration of DA sensor

Figure 5 displays the typical amperometric response of the biosensor based on the EDTAMWCNT for successive additions of DA under the optimal experimental conditions. The sensor exhibits a rapid and sensitive response to the addition of DA. The current response of the sensor is less than $1 \mathrm{~s}$ among the calibration range of DA.

The researches reported that the sensors based on the integration of CNTs and some other materials can enhance the eletrocatalytic activity and improve the detection sensitivity remarkably by utilizing their synergic action [22-25]. The previous work exhibited that the response current of the GCE/AuNPs and GCE/MWCNTs sensors [17, 19-21] were linear in the range from $10^{-6}$ to $10^{-4} \mathrm{~mol} . \mathrm{L}^{-1}$, the detection limit was at the level of $10^{-6}$ to $10^{-7} \mathrm{~mol} . \mathrm{L}^{-1}$. In the present research, the integration of AuNPs and EDTA-MWCNTs exhibits higher electrochemical catalytic activity due to their synergistic effect. The DA sensor based on the AuNPs and EDTA-MWCNTs matrixes shows wider linear current response and more sensitive detection limit as seen from Figure 6.

Figure 6 shows that the response current of the GCE/AuNPs/EDTA-MWCNTs sensor is linear in the range from $1.0 \times 10^{-8}$ to $6.0 \times 10^{-6}$ mol. $\mathrm{L}^{-1}$ with a detection limit of $3.0 \times 10^{-9}$ mol. $\mathrm{L}^{-1}$ based on signal/noise $=3$ and a correlation coefficient of $0.9948(n=9)$. Compared with GCE/AuNPs and GCE/MWCNTs sensors, the sensitivity of GCE/AuNPs/EDTA-MWCNTs sensor improved significantly. The sensitivity of the biosensor to DA can also be calculated from the linear region of calibration curve to be $1020 \mu$ A.L.mol ${ }^{-1} \cdot \mathrm{cm}^{-2}$.

The presence of AA $\left(40 \mu \mathrm{mol} . \mathrm{L}^{-1}\right)$ and uric acid $\left(3 \mu \mathrm{mol} . \mathrm{L}^{-1}\right)$ in the solution has a little interference with the detection of DA $\left(20 \mathrm{nmol} . \mathrm{L}^{-1}\right)$ with current increasing by $3.5 \%$. It is known that AA and UA exist in anionic form under the test conditions. At pH 7.2, EDTA exists in its carboxylate anions forms. Hence, there is plentiful negative charge on the surface of the sensor. By the benefiting from the charge repelling effect of EDTA-MWCNTs, the biosensor 
protects DA from the interference of AA and UA. The results show that the biosensor can substantially eliminate the interference from AA and UA.

The stability of the biosensor was investigated by amperometric measurements in the presence of $1.0 \mu \mathrm{mol} . \mathrm{L}^{-1} \mathrm{DA}$. The biosensor was tested every other day during 3 months and the sensitivity of the electrode response maintained over $90 \%$ of the original value. The result showed an excellence stability, long lifetime and high sensitivity of the sensor.

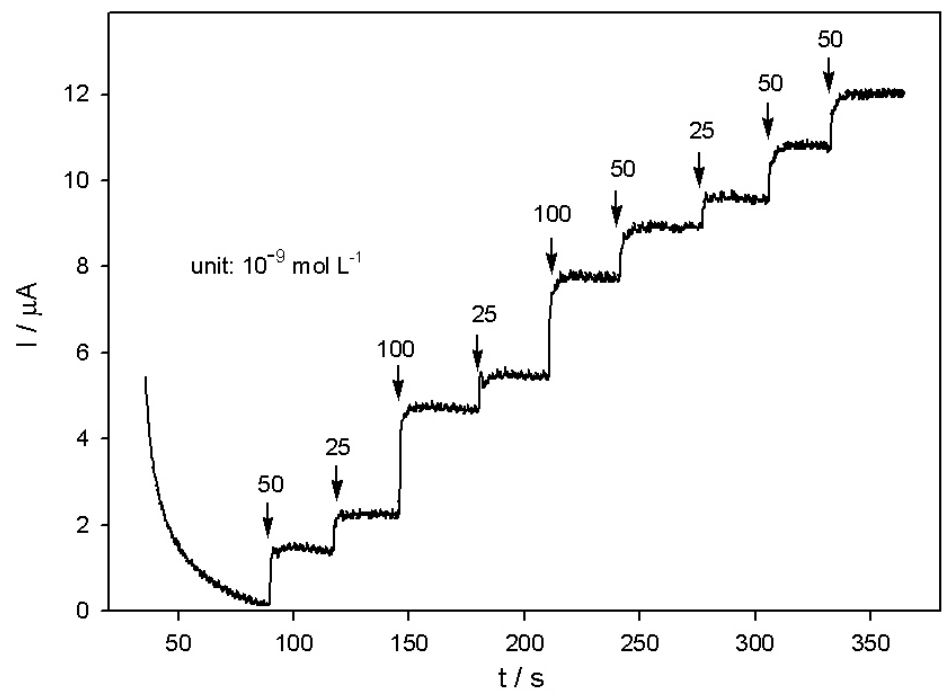

Figure 5. Current-time curve of the AuNPs and EDTA-MWCNTs modified GEC electrode toward successive addition dopamine at $0.280 \mathrm{~V}$.

Determination of DA in human urine

The determination of DA in human urine samples was performed using the propose method. Current response was recorded in the diluted urine samples and those spiked with DA. As shown in Table 1, the recoveries are satisfactory.

Table 1. Determination of the DA in urine.

\begin{tabular}{|c|c|c|c|}
\hline Human urine samples & Spiked $\left(\mathrm{nmol} . \mathrm{L}^{-1}\right)$ & Found $\left(\mathrm{nmol} . \mathrm{L}^{-1}\right)$ & Recovery (\%) \\
\hline \multirow{3}{*}{1} & 0 & 0 & \\
\cline { 2 - 4 } & 25.0 & 26.8 & 107.2 \\
\cline { 2 - 4 } & 50.0 & 53.2 & 106.4 \\
\hline \multirow{3}{*}{2} & 0 & 0 & \\
\cline { 2 - 4 } & 100.0 & 103.9 & 103.9 \\
\cline { 2 - 4 } & 150.0 & 156.2 & 104.1 \\
\hline \multirow{3}{*}{3} & 0 & 0 & 105.1 \\
\cline { 2 - 4 } & 80.0 & 84.1 & 104.8 \\
\cline { 2 - 4 } & 220.0 & 230.5 & 104.3 \\
\cline { 2 - 4 } & 0 & 0 & 103.6 \\
\cline { 2 - 4 } & 200.0 & 208.6 & \\
\hline \multirow{3}{*}{4} & 300.0 & 310.9 & \\
\end{tabular}

Bull. Chem. Soc. Ethiop. 2009, 23(3) 

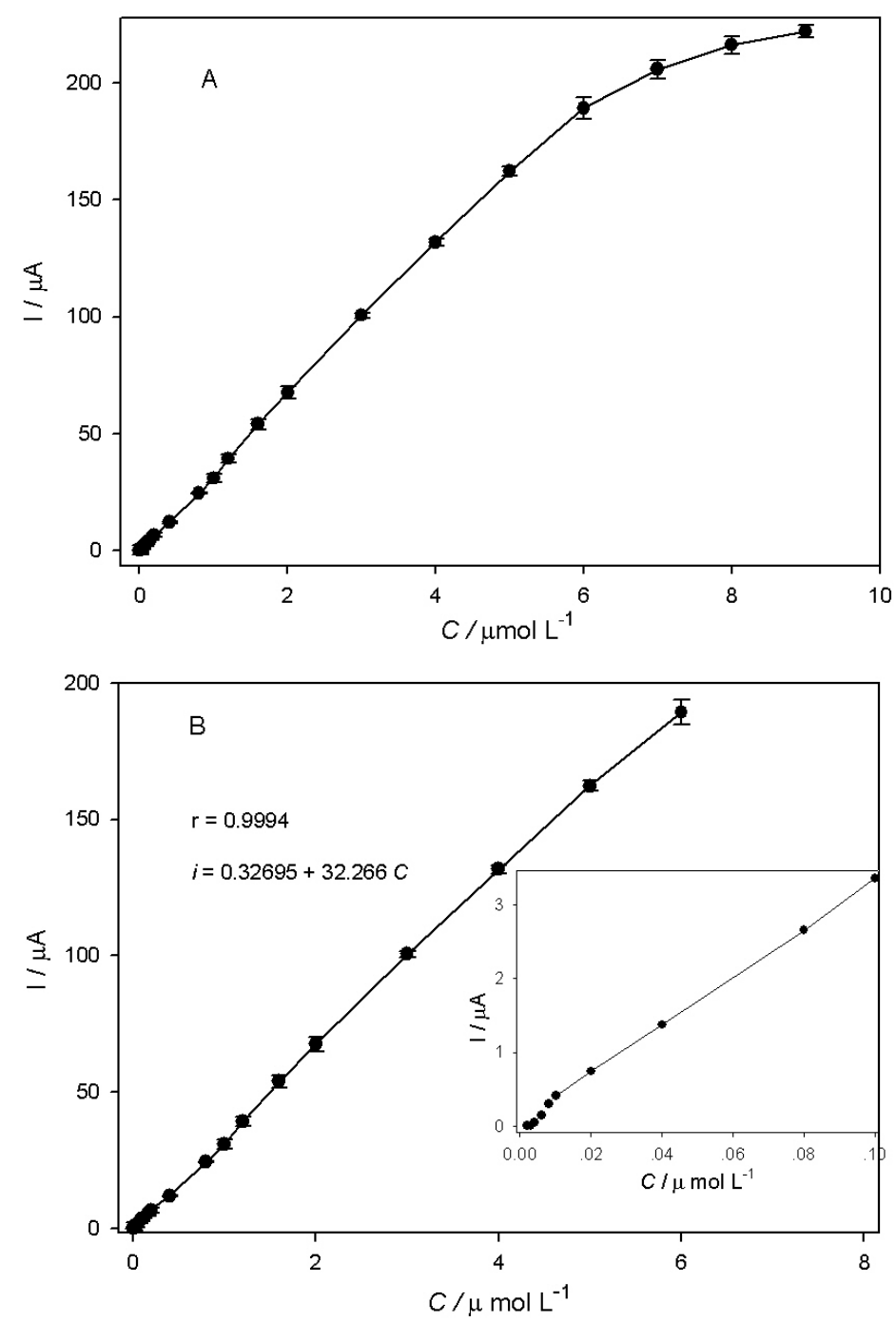

Figure 6. (A) The dependence of current response of sensor modified with AuNPs and EDTAMWCNTs on concentration of dopamine in 0.10 mol. $\mathrm{L}^{-1}$ PBS solution ( $\mathrm{pH} 7.2$ ), working potential: $0.280 \mathrm{~V}$. (B) The calibration curve in the range from $1.0 \times 10^{-8}$ to $6.0 \times 10^{-6} \mathrm{~mol} . \mathrm{L}^{-1}$. Inset shows the detection limit of $3.0 \times 10^{-9} \mathrm{~mol} . \mathrm{L}^{-1}$.

\section{CONCLUSIONS}

In this work, EDTA-MWCNT nanohybrids were prepared by grafting EDTA onto carboxylated multi-walled carbon nanotubes. A high selective and sensitive DA sensor was fabricated by covalently modified GCE with AuNPs and EDTA-MWCNTs nanomaterials benefited from the synergic effect of AuNPs and EDTA-MWCNTs. The EDTA-MWCNTs with its electronegative charge repelling effect has been utilized to eliminate the interference caused by AA and UA for

Bull. Chem. Soc. Ethiop. 2009, 23(3) 
the DA detection. It provides an alternative system with excellent sensitivity and antiinterference ability. The detection limits were down to 3 nanomolar concentration. Because the EDTA-MWCNT nanohybrids can also load many other biomaterials and expands the application scope of CNTs and holds great promise for biosensor development.

\section{AKNOWLEDGEMENTS}

The authors gratefully acknowledge financial support from the National Natural Science Foundation (Grants 20803020), and the Hunan Provincial Natural Science Foundation (Grant 04JJ30047, 07JJ6027).

\section{REFERENCES}

1. Zou, G. Basic Nerve Pharmacology, Science Press: Beijing; 1999; p 203.

2. O'Neill, R.D. Analyst 1994, 119, 767.

3. Zhang, X.B.; Guo, C.C.; Jian, L.X.; Shen, G.L.; Yu, R.Q. Analyst 2000, 125, 2285.

4. Zen, J.M.; Hsu, C.T.; Hsu, Y.L.; Sue, J.W.; Conte, E.D. Anal. Chem. 2004, 14, 4251.

5. Zeng, Y.L.; Li, C.X.; Tang, C.R.; Zhang, X.B.; Shen, G.L.; Yu, R.Q. Electroanalysis 2006, $18,440$.

6. de Toledo, R.A.; Santos, M.C.; Cavalheiro, E.T.G.; Mazo, L.H. Anal. Bioanal. Chem. 2005, $381,1161$.

7. Domenech, A.; Garcia, H.; Domenech-Carbo, M.J.; Galletero, M.S. Anal. Chem. 2002, 74, 562.

8. Mathiyarasu, J.; Senthilkumar, S.; Phani, K.L.N.; Yegnaraman, V. J. Appl. Electrochem. 2005, 5, 513.

9. Zhang, Y.Z.; Jin, G.Y.; Wang, Y.L.; Yang, Z.S. Sensors 2003, 3, 443.

10. Shang, F.; Liu, Y.; Hrapovic, S.D.; Glennon, J.; Luong, J H.T. Analyst 2009, 134, 519.

11. Joshi, P.P.; Merchant, S.A.; Wang, Y.; Schmidtke, D.W. Anal. Chem. 2005, 10, 3183.

12. Zeng, Y.L.; Huang, Y.F.; Jiang, J.H.; Zhang, X.B.; Tang, C.R.; Shen, G.L.; Yu, R.Q. Electrochem. Commun. 2007, 9, 185.

13. Huang, M.H.; Jiang, H.G.; Qu, X.H.; Xu, Z.A.; Wang, Y.L.; Dong, S.J. Chem. Commun. 2005, 44, 5560.

14. Jeong, S.; Jo, H.; Bae, S.R.; Jeon, S. Microchem. J. 2008, 88, 1.

15. Jiao, S.; Li, M.; Wang, C.; Chen, D.; Fang, B. Electrochim. Acta 2007, 52, 5939.

16. Wang, H-S.; Li, T-H.; Jia, W-L.; Xu, H-Y. Biosens. Bioelectron. 2006, $22,664$.

17. P. Zhang, F.H. Wu, G.C. Zhao, X.W. Wei Bioelectrochemistry 2005, 67, 109.

18. Zhang, M.N.; Gong, K.P.; Zhang, H.W.; Mao, L.Q. Biosens. Bioelectron. 2005, 20, 1270.

19. Zhang, L.; Jiang, X. J. Electroanal. Chem. 2005, 583, 292.

20. Wang, A-J.; Xu, J-J.; Zhang, Q.; Chen, H-Y. Talanta 2006, 69, 210.

21. Zhao, Y.; Gao, Y.; Zhan, D.; Liu, H.; Zhao, Q.; Kou, Y.; Shao, Y.; Li, M.; Zhuang, Q.; Zhu, Z. Talanta 2005, 66, 51.

22. Zhang, M.G.; Gorski, W. J. Am. Chem. Soc. 2005, 127, 2058.

23. Quinn, B.M., Dekker, C., Lemay, S.G., J. Am. Chem. Soc. 2005, 127, 6146.

24. Hrapovic, S.; Liu, Y.L.; Male, K.B.; Luong, J.H.T. Anal. Chem. 2004, 76,1083.

25. Yang, M.; Yang, Y.; Liu, Y.; Shen, G.; Yu, R. Biosens. Bioelectron. 2006, 21, 1791.

26. Yu, R.Q.; Chen, L.W.; Liu, Q.P.; Lin, J.Y.; Tan, K.L.; Siu Choon Ng, H.; Chan, S.O.; Xu, G.Q.; Hor, T.S.A. Chem. Mater. 1998, 10, 718.

27. Jiang, K.Y.; Eitan, A.; Schadler, L.S.; Ajayan, P.M.; Siegel, R.W. Nanoletters 2003, 3, 275.

28. Frens, G. Nature Phy. Sci. 1973, 241, 20.

29. Grabar, K.C.; Freeman, R.G.; Hommer, M.B.; Natan, M.J. Anal. Chem. 1995, 67, 735.

30. Venton, B.J.; Wightman, R.M. Anal. Chem. 2003, 75, 414A. 